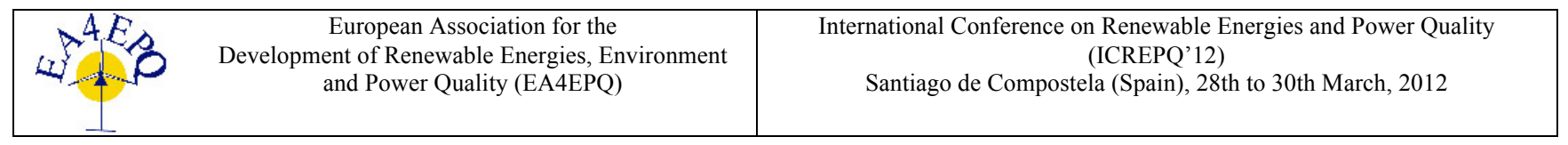

\title{
Advanced tools for electricity consumption monitoring
}

\author{
M. Domínguez, J.J. Fuertes, S. Alonso, M.A. Prada, A. Morán, P. Barrientos \\ SUPPRESS Research Group, University of León \\ Escuela de Ingenierías \\ Campus de Vegazana, León, 24007, (Spain) \\ Phone/Fax number: +0034 987 291694, e-mail: manuel.domínguez@unileon.es
}

\begin{abstract}
In this work, the power monitoring system developed at the University of León is presented. This system includes both traditional and advance monitoring tools based on statistical and data mining techniques. These techniques exploit data in order to search electrical patterns, detect anomalies, predict future consumption, optimize power, etc. Some results obtained from the monitoring system during year 2010 are presented.
\end{abstract}

\section{Key words}

Electricity consumption, monitoring tools, electrical models, Self-Organizing Map (SOM), Isometric Feature Mapping (ISOMAP).

\section{Introduction}

In today's society, where electricity consumption has experienced a significant increase in the last years, it is necessary to carry out actions which promote saving and energy efficiency for the sake of a more sustainable development [1]. One of these actions must be the introduction of electrical monitoring systems that measure, store and exploit the available information in electrical data to define responses aimed at improving energy efficient [2]. Currently, these systems are scarce and inefficient because they are only focused on the storage of few electrical variables, mainly, those involved in billing. Frequently, this storage is made with a low sampling period, which imply the loss of certain information about the system dynamics.

Therefore, it is necessary to improve the design and development of electrical monitoring systems. The modern monitoring systems should be based on open and non-proprietary architectures that use standard technologies to facilitate the full integration with other systems [3]. Besides providing traditional monitoring tools, such as visualization of instantaneous values, trends, alarms based on thresholds, minimum and maximum values, etc., these systems should include advanced monitoring tools that exploit the enormous amount of information available in data. These tools should be based on statistics and data mining techniques to provide a better understanding of the system and improve decision making. Finally, a vital requirement in modern monitoring systems is the remote access through the Internet. This way, monitoring can be done from anywhere, with an Internet connection. In this work, advanced monitoring tools for electrical power have been developed. These tools have been used to monitor the electrical system in the University of León.

This paper is organized as follows: section 2 describes the architecture of the monitoring system. Section 3 presents the advanced monitoring tools included in the system. Experimental results are exposed in section 4. Finally, conclusions are listed in section 5 .

\section{Architecture of the monitoring system}

The Campus of the University of Leon is composed of a group of buildings used for teaching, research or to provide complementary support services: cafés, data centers, administrative buildings, etc. The electrical power consumption in these buildings, framed in the tertiary sector, corresponds basically to lighting systems, heating, ventilating and air-conditioning systems (HVAC), as well as industrial-type equipment associated with the research centers. In Fig. 1 and Table I, all the buildings and billing points of the Campus can be observed.

The architecture adopted for the monitoring system of electrical power in the buildings of the University of León is based on a three-layer structure [4]. This structure follows the client-server pattern, where an intermediate layer is added to improve the modularity and the scalability. This layer manages the information provided by the server-layer and consists of a database.

The Server layer includes the electrical meters and the data acquisition server. This server records data from the meters and store them orderly in the database. The Middle layer is formed by the storage server, the data mining server and the web server. The storage server is composed basically of a relational database and its management system (DBMS). 


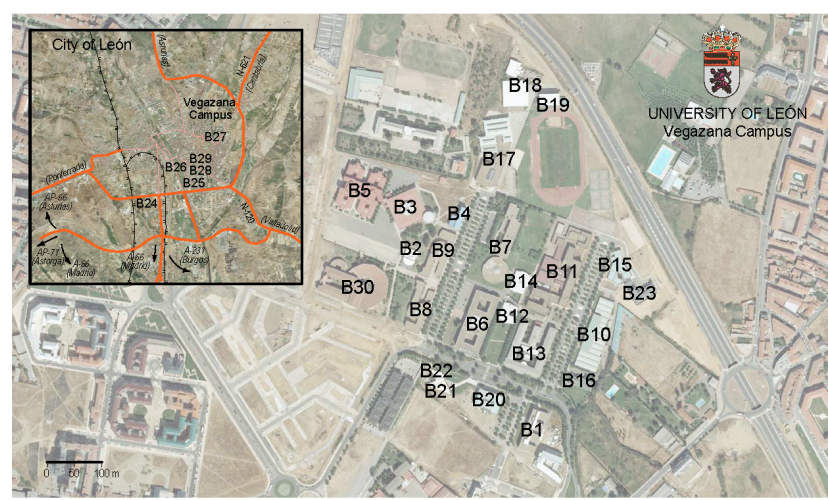

Fig. 1. Buildings of the University of León.

The Client-layer contains the monitoring interface to be presented to the analysts. The system architecture allows any computer with Internet access to be used for monitoring. The monitoring system is accessible from both the University of León and outside. The web applications developed to monitor the system run on the web server. These applications display common dynamic elements such as charts, maps, controls, indicators, etc. which define the user interface for monitoring. The technologies used to develop the graphical interfaces are HTML, ASP.NET [5] and LabVIEW [6]. In the next section, those tools are described in detail.

\section{Monitoring tools}

In this section, the developed tools to supervise, manage, and optimize the power consumption at the University of León are presented. These tools can be classified into two groups: traditional and advanced monitoring. At the same time, a new classification can be made in each of these groups depending on whether the tool is used to on-line or off-line monitoring.

\section{A. Traditional monitoring tools.}

These tools allow the visualization of the electrical variables recorded by each meter. The information is presented as charts, plotted against time. Using these tools, the analyst can observe, in real time, the instantaneous value of the variables and receive alarms or warnings while monitoring in on-line mode. Additionally, in off-line mode, these tools can retrieve historical data to visualize and analyze maximum and minimum values, trends of electrical variables, etc., always with respect to time. In Fig 2, the web-monitoring environment, which integrates these traditional tools to on-line monitoring, is showed. The web page is structured into different tabs for voltages, currents, powers, harmonics, THD, etc. Inside of them, temporary charts are shown as a function of time. The meter/building is selected through a dropdown menu.

\section{B. Advanced monitoring tools.}

These tools analyze data using statistical and data mining techniques to obtain electrical models [7] [8]. These models allow the analyst to reason using the knowledge extracted from data, and make decisions to optimize the system. The advanced monitoring tools are divided into different groups and, as in the previous case, can be used for on-line and off-line monitoring.

Table I. - Buildings and billing points of the electrical system

\begin{tabular}{|c|c|c|}
\hline $\begin{array}{l}\text { BILLING } \\
\text { POINTS }\end{array}$ & BUILDINGS & DESCRIPTION \\
\hline $\mathrm{P} 1$ & B1 & Data Center \\
\hline \multirow{2}{*}{$\mathrm{P} 2$} & B2 & Radio \\
\hline & $\mathrm{B} 30$ & Business School \\
\hline \multirow{6}{*}{ P3 } & B3 & Engineering School I \\
\hline & B4 & Lecture Hall \\
\hline & B5 & Engineering School II \\
\hline & B17 & School of Sports \\
\hline & $\mathrm{B} 18$ & Sports Center I \\
\hline & B19 & Sports Center II \\
\hline \multirow{4}{*}{ P4 } & B6 & School of Arts \\
\hline & B7 & Law School \\
\hline & B8 & School of Education \\
\hline & B9 & School of Labor Studies \\
\hline \multirow{7}{*}{ P5 } & $\mathrm{B} 10$ & Veterinary Hospital \\
\hline & B11 & Veterinary School \\
\hline & $\mathrm{B} 12$ & Cafeteria I \\
\hline & B13 & Biology School \\
\hline & B14 & Service Center \\
\hline & B15 & Farm Development \\
\hline & B16 & Animal Facilities \\
\hline \multirow{3}{*}{ P6 } & $\mathrm{B} 20$ & Central Library \\
\hline & B21 & Bank \\
\hline & B22 & Cafeteria II \\
\hline P7 & B23 & Molecular Biology Research Center \\
\hline P8 & B24 & Agricultural Facilities \\
\hline $\mathrm{P9}$ & $\mathrm{B} 25$ & Dormitory \\
\hline $\mathrm{P} 10$ & B26 & Administration Offices \\
\hline P11 & B27 & Environmental Research Center \\
\hline $\mathrm{P} 12$ & B28 & Mining Engineering School \\
\hline $\mathrm{P} 13$ & B29 & Language Center \\
\hline
\end{tabular}

Visualization and comparison tools: these tools use projection techniques to achieve dimensionality reduction and vector quantization [9], such as the Self-Organizing Map (SOM) [10] and Isometric Feature Mapping (ISOMAP) [11]. These techniques have been used in the data analysis which will be exposed in section 4 . Nevertheless, other similar techniques can be used as well. The comparison tools aim to project onto $2 \mathrm{D}$ spaces the electrical power profiles from buildings. These projections can be used to compare and facilitate the visualization building groups. The relative distance between points indicates the similarity/difference in the electrical behavior of buildings. If two points are close, then the electrical profile of corresponding buildings is similar.

The Self-Organizing Map (SOM) [10] is a neural network that produces a nonlinear ordered mapping from a highdimensional input space onto a low-dimensional visualization lattice, which captures the latent structure of the data. The algorithm follows a competitive-cooperative scheme which, iteratively, computes the Best Matching Unit (BMU) $\mathbf{m}_{c}$ that is the closest to the current sample $\mathbf{x}_{k}$ 
$c=\arg \min _{i}\left\|\mathbf{x}_{k}-\mathbf{m}_{i}(t)\right\|$

and adapts it and its neighboring units to further approximate that sample,

$$
\mathbf{m}_{i}(t+1)=\mathbf{m}_{i}(t)+\alpha(t) h_{c i}(t)\left[\mathbf{x}_{k}-\mathbf{m}_{i}(t)\right]
$$

where $\alpha(t)$ is a learning factor and $h_{c i}(t)$ is a Gaussian neighborhood function, both of them monotonically decreasing with time.

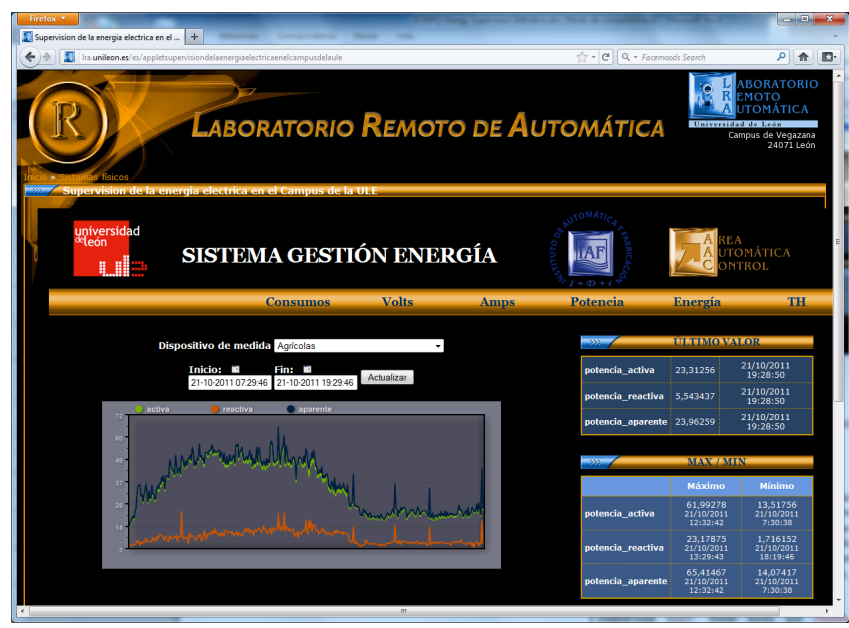

Fig. 2. Web-based monitoring system of the electrical variables at the University of León.

The SOM has been successfully applied as a data visualization tool in many fields, since it creates an ordered and compact low-dimensional representation of the data structure [12]. For instance, any meaningful variable can be represented in the visualization space using a color value with the component planes. These planes present information in a consistent way and, therefore, they can be compared and explored concurrently. SOM has previously been applied in the field of power monitoring, yielding interesting and promising results [13].

ISOMAP [11], as well as other multidimensional scaling (MDS) algorithms, aims to preserve dissimilarities of data in an output low-dimensional space through the minimization of a cost function. Unlike the classical MDS, which uses Euclidean distance, ISOMAP uses geodesic distance. The geodesic distance is defined as the shortest path along a manifold which joins two of its points. It is more suitable for dimensionality reduction than Euclidean distance, since points which are deceptively close on the high-dimensional input space might be far apart on the underlying manifold. The algorithm has three steps: first, a graph is built connecting each point with its $\mathrm{K}$ nearest neighbors; later, the shortest paths are computed; finally, a classical MDS is applied to the matrix of graph distances.

Prediction tools: the generation of electrical models from past behavior makes it possible to forecast future electrical behavior. The prediction tools have been developed using projection techniques based on SOM [14]. Since the SOM can be seen as a non-linear regression, the representation can be used for predicting, in this case, the power variables can be predicted using the environmental ones. It is worth noting that the codebook vectors represent local averages of the training data. Regression is accomplished by searching the BMU, which is calculated as:

$$
c=\arg \min _{i} \sum_{j \in S}\left(\mathbf{x}_{k}-\mathbf{m}_{i j}\right)^{2}
$$

where $S$ is the set of known vector components, in this case the environmental conditions of the sample. As an output, a copy of components $k \notin S$ of the codebook vector $\mathbf{m}_{i}: \hat{y}=m_{c k}$, which correspond to the predicted power variables, are given. The accuracy of the model is controlled by two factors. The first one is the number of codebook vectors, since a SOM with large number of map units allows a densely quantization of the data space and enables an accurate regression. The second one is the accuracy of the prediction of the environmental variables, since a bad estimation of the environmental conditions for a particular day will forecast power consumption which won't correspond with that particular day.

The tools can be used to predict consumption in buildings in short (1-6 hours) and medium terms (days). In the first case, we can predict the load distribution and so, try to compensate it. In the second case, the obtained information gives us an advantageous position with respect to the electricity supplier in the price negotiation phase.

Optimization tools: these tools are used to determine the optimum power to contract, considering three billing periods, Peak, Flat and Off-Peak. Electric companies distribute the electricity consumed in these three periods, taking into account the hour of day, day of week and season of the year. The power demand and energy consumed in each period have a different cost. The optimization tools determine the optimum power to be contracted using the method of cost function minimization. Using Matlab software, power-cost functions for each period are obtained. The functions compute the annual cost with respect to power from data of past periods. Then, the minimums of these functions are calculated. These minimums are the optimum powers for each period.

\section{Data analysis and results}

In this section, results from the application of the monitoring tools are presented. The monitoring system presented in this paper has been introduced during the year 2009. Data recorded during the year 2010 have been used in this data analysis. Data have been sampled at 2 minutes. We present three different analyses: determination of optimum power, buildings comparison with regard to their daily consumption profiles and weekly analysis of electrical variables in the buildings. 


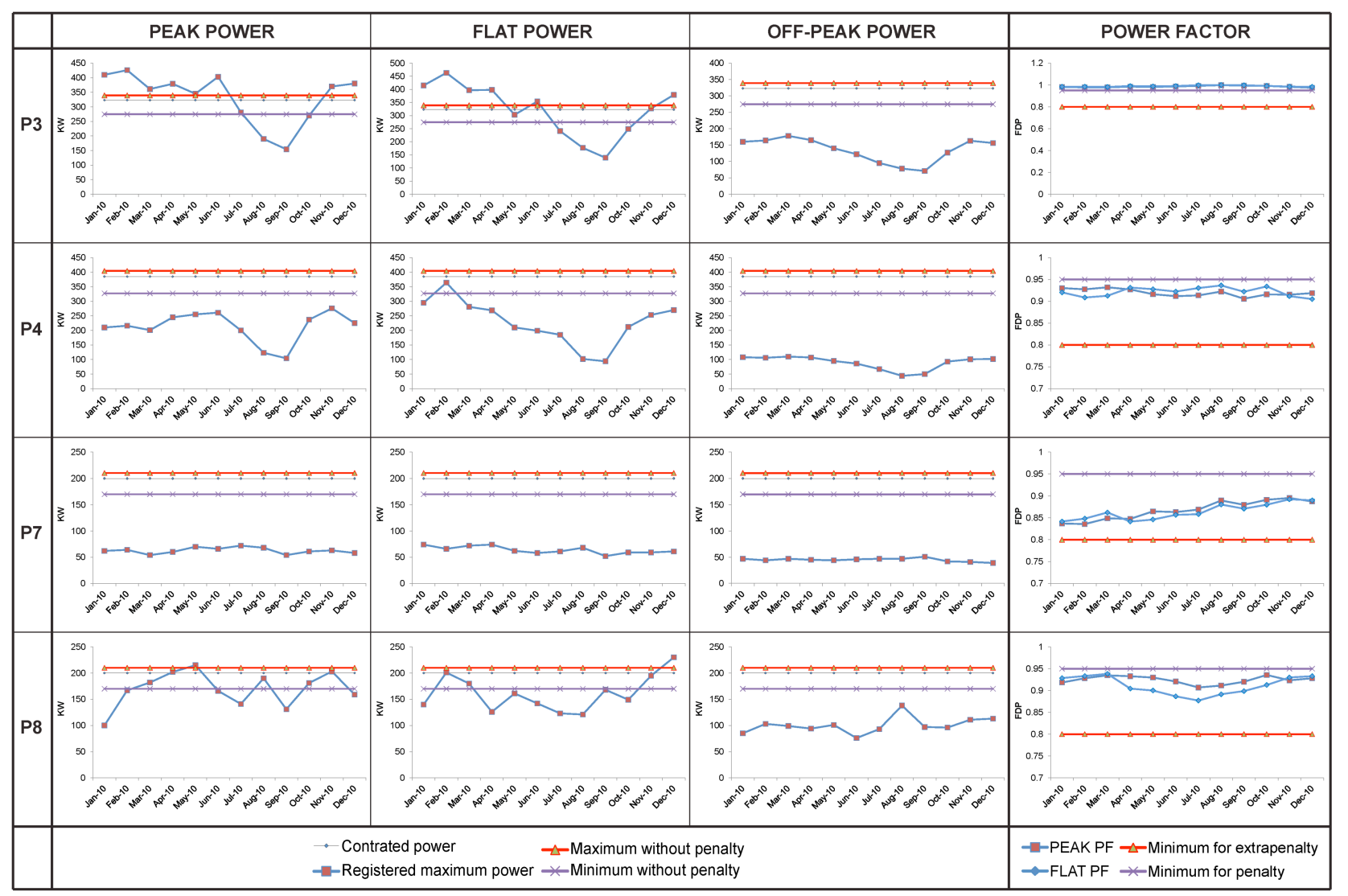

Fig. 3. Power analysis in billing points $\mathrm{P} 7, \mathrm{P} 4, \mathrm{P} 7$ and $\mathrm{P} 8$.

\section{A. Determination of optimum power.}

A first result from data analysis is that the electric supplier penalizes the University due to the difference between the contracted and consumed power. In Fig. 3, the maximum power and the power factor, recorded every month during the year 2010, has been plotted for the three billing periods (Peek, Flat and Off-Peak), in four measurement points. The band with no penalization has also been plotted in every graph. Finally, the optimum power to hire has been calculated using the optimization tool.

Let us take, for instance, the graphs of the Molecular Institute (P7). This billing point has a contracted power of $200 \mathrm{~kW}$ for the three periods in 2010. However, the consumed power is about $100 \mathrm{~kW}$, well below the minimum without penalty (about $170 \mathrm{~kW}$ ). This involves an active power penalty. Regarding power factor, its value ranges between 0.83 and 0.89 , involving a reactive energy penalty. Therefore, it is necessary to increase the power factor and decrease the contracted power in this building. The estimated optimum power is $68 \mathrm{~kW}$ in this case.

It can be observed that the demanded power is also out of the band, in whole or in part, in the other billing points. Table II shows the differences between the contracted power and the optimum power for all the billing points, calculated by the optimization tool. Moreover, savings resulting from this action are shown. This analysis, performed for all the buildings, has helped to determine the causes of the penalties, which have been corrected the next year (2011).
Table II. - Optimum power and resulting saving

\begin{tabular}{|c|c|c|c|}
\hline $\begin{array}{c}\text { BILLING } \\
\text { POINT }\end{array}$ & $\begin{array}{c}\text { CONTRACTED } \\
\text { POWER (kW) }\end{array}$ & $\begin{array}{c}\text { OPTIMUM } \\
\text { POWER (kW) }\end{array}$ & $\begin{array}{c}\text { SAVING } \\
(\boldsymbol{\epsilon})\end{array}$ \\
\hline P2 & 145 & 137 & 91.92 \\
\hline P3 & 380 & 323 & 1011.51 \\
\hline P4 & 385 & 249 & 3422.76 \\
\hline P6 & 200 & 134 & 1665.77 \\
\hline P7 & 200 & 68 & 4441.75 \\
\hline P8 & 200 & 191 & 91.48 \\
\hline P9 & 60 & 45 & 200.4 \\
\hline P10 & 50 & 44 & 42.6 \\
\hline P11 & 35 & 41 & 46.8 \\
\hline P12 & 50 & 28 & 378.83 \\
\hline P13 & 35 & 58 & 428.56 \\
\hline
\end{tabular}

\section{B. Visualization of electrical variables based on SOM.}

The aim of this analysis is to monitor the electrical variables of the buildings by means of component planes from SOM. Real past data acquired and stored by the monitoring system are used for training a SOM. The selected data are from building B6 and correspond to 1 month (March, 2010) sampled at 2 minutes, i.e., the size of data set is 22320 samples. Nevertheless, data from other time periods or buildings could be perfectly used. Among all acquired variables, only nine of them were 
chosen to be visualized: weekdays, average of voltages, average of currents, active power, reactive power, power factor, neutral current, frequency and average of THD in currents. The weekdays are very useful to reach an organization of the SOM around this variable, making it possible to monitor of electrical variables according to weekdays in the buildings.

Before training, data were preprocessed to ignore erroneous samples and normalized to 0-1 range. A SOM, whose dimensions are $20 \times 30$ (600 units), was trained using the explained data set. The training epochs were 100 , the learning rate decreased in time, the neighborhood function was implemented as Gaussian, and the initialization was linear along the greatest eigenvectors. In the SOM training, a weight was given to the weekday variable to obtain the desired organization. In this scenario, the SOM models the electrical behavior of the buildings, taking into account the weekdays.

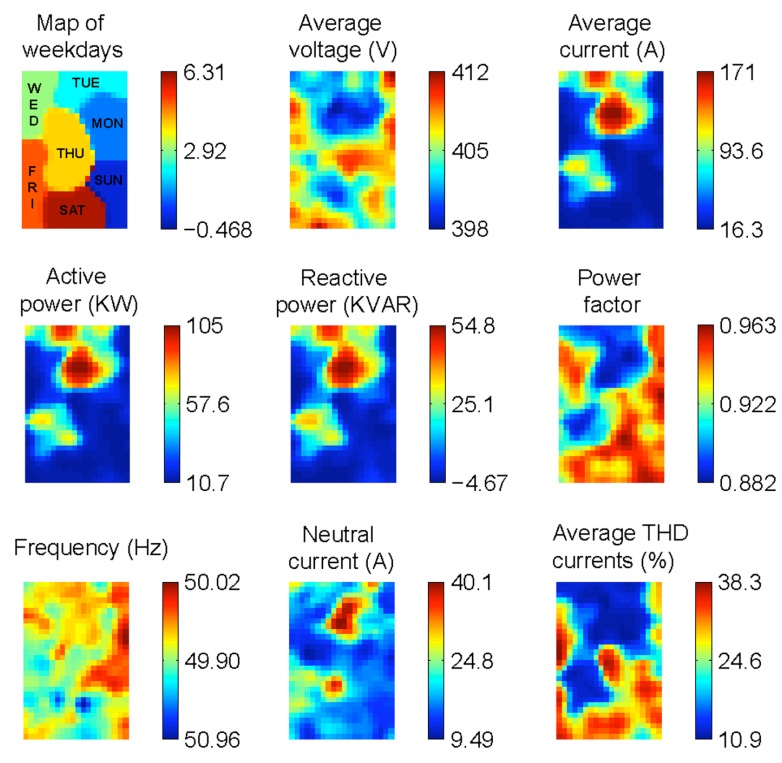

Fig. 4. Visualization of electrical variables based on Self Organizing Map (SOM).

As a result, component planes of the SOM are shown in Fig. 4. In the first component plane, we can observe 7 different zones corresponding to the weekdays. Note that the organization of the remaining components is conditioned on this variable. The variations of the average voltage are lower or equal to $3 \%$. As expected, the voltage decreases when the average current or active power increases, and is independent on the weekdays. There exists a strong correlation between average current and active power, as expected. Most of the active power demand happens from Monday to Thursday. These days contain small areas where the active power is very low, corresponding to the evenings and nights. On Saturday and Sunday, only a little residual power is demanded all day. The plane of the reactive power seems similar to the planes of the average current and active power. However, the plane of the power factor reveals that the relationship between active and reactive power is not linear. When active power is high, the power factor is low and vice versa. The highest values of power factor correspond to the weekends and the nights of the working days. It suggests that the equipment for power factor correction should be checked to avoid penalties in billing.

The frequency has no dependence on the weekday since this variable, likewise average voltage, is determined by electricity supplier. The plane of neutral current suggests that most of the electrical loads in the building are 1phase, provoking a current unbalance when active power increases. Regarding the average THD of currents, it can be pointed that, surprisingly, high values of this variable take place on the weekend and nights, i.e., periods with a little active power demand. It can be due to some constant power electronic system, such as the uninterruptible power supply, which distorts the current wave.

\section{Building comparison according to their power profiles.}

The aim of this analysis is to compare all buildings according to their daily power profiles. When there are many buildings, it is difficult to compare power demand among all of them for a certain time. Projection methods can facilitate this task, reducing the dimensionality of data and representing each building as a point on a $2 \mathrm{D}$ graph. In this case, active power data, acquired and stored by the monitoring system, are projected using ISOMAP mapping. For the sake of clarity of the projections, only half of the buildings are used in the analysis. The selected buildings were B1, B6, B7, B9, B10, B11, B12, B13, B16, $\mathrm{B} 18, \mathrm{~B} 19, \mathrm{~B} 23, \mathrm{~B} 24, \mathrm{~B} 25$ and $\mathrm{B} 30$. The size of data set is 22320 samples, i.e., 1 month (October, 2010) sampled at 2 minutes. Nevertheless, power data from other time periods or buildings could be perfectly used.

In a previous step, power data were filtered by means of a moving average with 15 samples to delete the signal noise. Next, power data were resampled down with a factor 15, i.e., 1488 samples to speed up the mapping. Finally before mapping, a linear interpolation was applied to substitute the missing values. To take into account the weekly periodicity in the power demand, an individual mapping for each weekday was carried out. Therefore, 7 different 2D graphs will be obtained. ISOMAP mapping was applied to the preprocessed data set using the parameter $\mathrm{k}=30$ neighbors. The dimension of the output space is 2 (2D visualization graph for each weekday). Other analogous methods such as Sammon's mapping and t-SNE were tried but ISOMAP provided better results, making comparison among buildings easy.

The obtained results are shown in Fig. 5. In a general and quick view, the difference between weekend and working days with regard to power demand can be observed. In the graph corresponding to the Saturday (and similarly on Sunday), most of the buildings are projected close together, unlike the graphs corresponding to the working days, where buildings are scattered. Every weekday, buildings B18 and B19 are projected together and far from the remaining buildings. It can be because of the fact that these two buildings are used for sport practice in the evening and weekend. On the contrary, most of the buildings are used for teaching and research in the morning and afternoon. Note that B25 is projected close to 
both buildings, B18 and B19, except on Thursday, Friday and weekend. It can be due to the habits of the students who stay overnight in the dormitory for these weekdays.

Buildings B6, B7, B9 and B12 are always located close together in the graphs, indicating that they have similar power profiles. The projection of the buildings B10, B11, $\mathrm{B} 13, \mathrm{~B} 16, \mathrm{~B} 23$ and B24 reveals that they have a common profile. In fact, all of these buildings are characterized by large area, sophisticated research-oriented machinery and high activity. It can be pointed out that B1 is not very far from the last building set and therefore, we can consider that they have a similar profile, although the residual power in B1 is higher, probably due to many servers in the data center. $\mathrm{B} 30$ is almost always located in the middle of two building sets (B6, B7, B9, B12 and B10, B11, B13, B16, B23, B24).
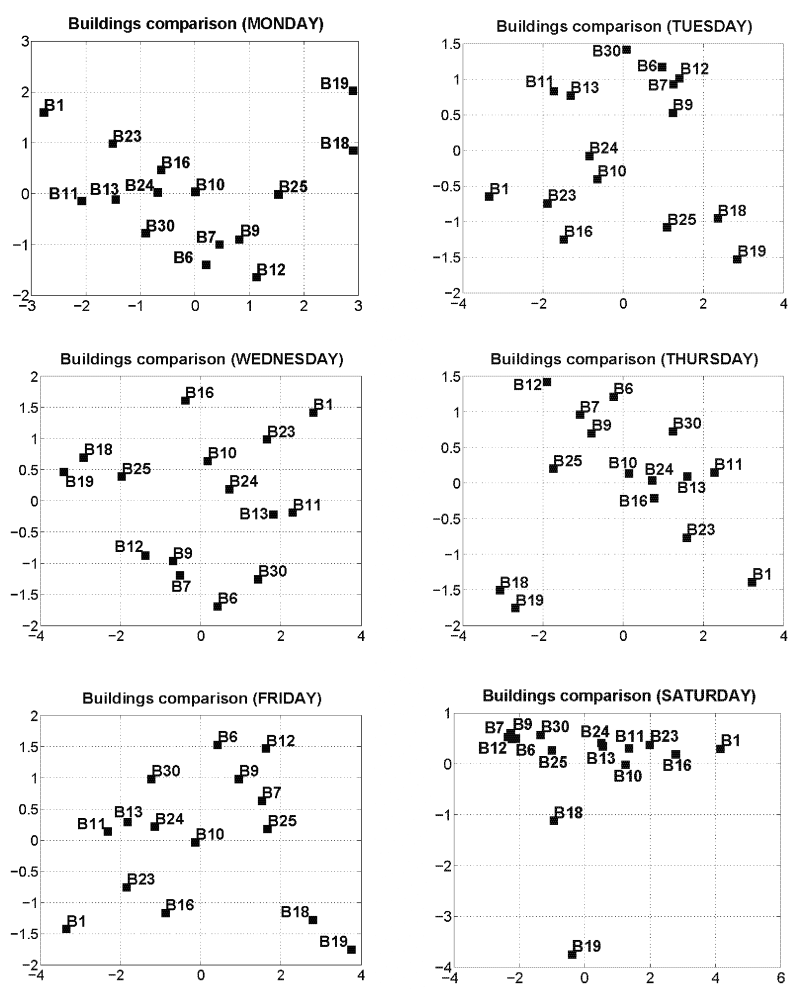

Fig. 5. Building comparison according to their power profiles for each weekday using ISOMAP mapping.

\section{Conclusions}

The use of advanced tools for electrical monitoring which rationalize electricity consumption is still a pending issue in society. Classical monitoring tools provide limited displays, in different formats, of current and historical values recorded by the meters and focus only on the variables involved in billing. Moreover, data are usually sampled at low frequency. In this paper, we have developed advanced monitoring tools which are not limited to display stored data but use statistical and data mining techniques to compare buildings according to their electrical profiles, predict consumption in a short or long term, detect deviations, or optimize the power to be contracted.

\section{Acknowledgement}

This work was supported in part by the Spanish Ministerio de Ciencia e Innovación (MICINN) and the European FEDER funds under grants DPI2009-13398-C02-01 and DPI2009-13398-C02-02.

\section{References}

[1] Gershenfeld, N., Samouhos, S. and Nordman, B. (2010), "Intelligent infrastructure for energy efficiency", Science, 327(5969), pp. 1086-1088

[2] European Parliament (2010), "Directive 2010/31/EU of the European Parliament and of the Council of 19 may 2010 on the energy performance of buildings (recast)", Official Journal of the European Union, 53(L153).

[3] Domínguez, M., Reguera, P., Fuertes, J. J., Díaz, I. and Cuadrado, A. A. (2007), "Internet-based remote monitoring of industrial processes using Self-organizing maps", Engineering Applications of Artificial Intelligence, 20(6), pp. 757-765.

[4] Eckerson, W.W. (1995). "Three tier client/server architectures: Achieving scalability, performance, and efficiency in client/server applications". Open Information Systems, 3(20), pp. $46-50$.

[5] Liberty, J. and Hurwitz, D., Programming ASP.NET, O'Reilly Media, USA (2003), 2nd ed., ISBN 0-59-600487-7.

[6] National Instruments, LabVIEW User Manual, USA (2003), April ed., no. 320999E-01.

[7] Bishop, C.M., Pattern recognition and machine learning. Information science and statistics, Springer New York (2006), 1st ed., ISBN 978-0-387-31073-2.

[8] Ferreira de Oliveira, M. C. and H. Levkowitz (2003), "From visual data exploration to visual data mining: A survey", IEEE Transactions on Visualization and Computer Graphics, 9(3), 378-394.

[9] Lee, J. A. and M. Verleysen, Nonlinear Dimensionality Reduction, Information Science and Statistics, Springer, (2007), ISBN 978-0387393506.

[10] Kohonen, T. Self-Organizing Maps, $3^{\text {rd }}$ edition, SpringerVerlag New York, Inc., Secaucus, NJ, USA (2001), ISBN 3 5406-7921-9.

[11] Tenenbaum, J. B., V. de Silva and J. C. Langford (2000), "A global geometric framework for nonlinear dimensionality reduction", Science, 290, 2319-2323.

[12] Vesanto, J. (1999), "SOM-based data visualization methods", Intelligent Data Analysis, 3(2), 111-126.

[13] Domínguez, M., J. Fuertes, I. Díaz, A. Cuadrado, S. Alonso and A. Morán (2011), "Analysis of electric power consumption using self-organizing maps", in proceedings of the 18th World Congress of the International Federation of Automatic Control, IFAC2011, Milano.

[14] Hollmén, J. and Simula, O. (1996), "Prediction models and sensitivity analysis of industrial production process parameters by using the Self-Organizing Map", in proceedings of IEEE Nordic Signal Processing, Espoo. 